\title{
Lecciones del programa de prevención temprana de la violencia, Medellín, Colombia
}

\author{
Luis Fernando Duque, ${ }^{1}$ José Fernando Orduz, ${ }^{1}$ \\ Juan de Jesús Sandoval, ${ }^{1}$ Beatriz Elena Caicedo ${ }^{1}$ \\ y Joanne Klevens ${ }^{2}$
}

Forma de citar

Duque LF, Orduz JF, Sandoval JJ, Caicedo BE, Klevens J. Lecciones del Programa de Prevención Temprana de la Violencia, Medellín, Colombia. Rev Panam Salud Publica. 2007;21(1):21-9.

RESUMEN Objetivo. Describir las características y la evolución del Programa de Prevención Temprana de la Violencia del Municipio de Medellín, Colombia, y evaluar los resultados de la primera etapa tres años después de su implementación.

Métodos. Se llevó a cabo un análisis anterior (año 2001) y posterior (año 2004) de cinco variables - sintomas de agresión directa, sintomas de agresión indirecta, sintomas de prosocialidad y rendimiento escolar - en una muestra por conveniencia de 339 niños y sus familias que habian participado en el programa.

Resultados. Se evidenciaron varios efectos positivos del programa. Se observó una disminución de los sintomas de agresión directa y de los de agresión indirecta, pero en este último caso sólo en niñas y en mayores de 12 años. Se evidenció asimismo un aumento en la prosocialidad en niños de todas las edades y de ambos sexos, incluso en los que presentaban menor grado de prosocialidad en 2001. También se observó en todo el grupo una mejora del rendimiento escolar. Estos resultados pueden haber sido afectados por algunas modificaciones en la implementación del programa de prevención y por la gran peligrosidad del barrio, que ocasionó una disminución de la frecuencia de las visitas domiciliarias a las familias.

Conclusiones. El programa parece tener efectos preventivos, puesto que se observa una disminución de la agresión directa en los niños con mayor nivel de agresión, y un efecto protector en el aumento de la prosocialidad en los niños menos agresivos.

Palabras clave Prevención primaria, violencia, agresión, rendimiento escolar bajo, eficacia, Colombia.

1 Universidad de Antioquia, Facultad Nacional de Salud Pública, Medellín, Colombia. Toda correspondencia deberá dirigirse a: Profesor Luis Fernando Duque, Universidad de Antioquia, Facultad Nacional de Salud Pública, Calle 62 \# 52-59, Ofic. 211, Medellín. Colombia; teléfono y fax: (57 4) 21068 68; correo electrónico: lfduque@une.net.co y lfduque@guajiros.udea.edu.co

2 National Center for Injury Prevention and Control, Centros para el Control y la Prevención de Enfermedades (CDC), Atlanta, Georgia, Estados Unidos.
Las muertes intencionales generan en el mundo el $3,4 \%$ de la carga de enfermedad, o sea, de los años de vida saludables perdidos (1), alrededor del $25 \%$ en Colombia (2) y el $44,8 \%$ en Medellín (3). Es decir, en Medellín se pierden casi tantos años de vida saludables por homicidio y suicidio que por todas las demás causas de incapacidad y muerte sumadas.

Además de muertes y lesiones físicas no mortales, la violencia también genera consecuencias psicológicas en los testigos y en las víctimas. En los niños puede ocasionar problemas de aprendizaje, malas relaciones 
interpersonales y comportamiento agresivo y antisocial (4).

La agresión temprana es uno de los mejores factores de pronóstico de agresión y criminalidad en la vida adulta (5-9), y es posible detectarla desde una época tan temprana en la vida como los tres años de edad (10).

Estudios de cohorte llevados a cabo en diferentes sitios han encontrado que los niños en que la agresión se manifiesta a temprana edad y persiste en la juventud o la adultez presentan concomitantemente otros problemas de comportamiento, como delincuencia, abuso de sustancias psicoactivas, conductas sexuales riesgosas, maternidad temprana, depresión y dificultades en la interrelación con otros (11-15). La existencia de estas comorbilidades sugiere que dichos comportamiento proceden de las mismas causas y, por ende, un programa de prevención temprana de la agresión puede tener efectos benéficos sobre ellos.

Aunque numerosas instituciones e investigadores en diferentes contextos culturales han llevado a cabo programas de prevención temprana de la agresión en niños, adolescentes y jóvenes, muy pocos de estos programas han sido objeto de evaluaciones de impacto por medio de estudios controlados al azar, y entre ellos, son contados los que han mostrado ser exitosos $(16,17)$. Muy pocas de las intervenciones que parecen haber sido efectivas para la prevención de la agresión han pasado de la fase piloto o de demostración a la fase de expansión a mayor escala en comunidades.

Un panel de expertos recientemente convocado por los Institutos Nacionales de Salud de los Estados Unidos (18) concluyó que las intervenciones exitosas para la prevención de la agresión tienden a compartir una constelación de características: a) se fundamentan en un marco teórico sólido, b) se orientan hacia factores de riesgo de gran peso, c) son de larga dura-ción (un año, por lo menos), d) siguen la estrategia cognitivoconductual $y$ son multimodales $y$ multicontextuales, e) fomentan o fortalecen las competencias sociales para promover habilidades en los jóvenes o en sus familias, f) son apropiadas para el desarrollo personal y comunitario, g) no se llevan a cabo en instituciones con ambiente coercitivo $y \quad f)$ tienen características y formas de ejecución que permiten o favorecen una buena adhesión al programa.

\section{El Programa de Prevención Temprana de la Violencia de Medellín ${ }^{3}$}

Se ha referido que en Medellín, alrededor del $13 \%$ de los niños escolares y preescolares presentan altos índices de agresividad y otros problemas de comportamiento (19). Otros estudios también han evidenciado que entre el 30 y el $84 \%$ de estos niños siguen presentando estos problemas de comportamiento durante la adolescencia y en la edad adulta $(20,21)$.

Consciente de esta situación, en 1998, el Municipio de Medellín inició una intervención denominada Programa de Convivencia Ciudadana, que constituye la iniciativa más importante y de mayor alcance que haya emprendido la ciudad para promover la convivencia ciudadana y prevenir y controlar la violencia.

Este programa tiene seis componentes, uno de los cuales es la prevención de la violencia en niños y jóvenes (22). Como parte de este componente nació en 2001 el Programa de Prevención Temprana de la Violencia, con objeto de disminuir los índices de agresión, propiciar el desarrollo de comportamientos prosociales y prevenir la violencia y la agresión, el consumo de sustancias psicoactivas y el bajo rendimiento escolar en los niños de entre 3 y 11 años de edad escolarizados en las instituciones educativas públicas de enseñanza preescolar y básica primaria del municipio de Medellín.

\footnotetext{
Agradecemos la asesoría de la Profesora Eryka Montoya por sus aportes para la redacción de este apartado.
}

El programa se diseñó en 2000, luego de consultas con diferentes instituciones de la ciudad, y se formuló con un enfoque sociocognitivo, basándose en dos estrategias: a) la enseñanza de habilidades prosociales en el aula y el manejo concomitante, consistente y no violento del comportamiento de los niños en la escuela por parte de los maestros, basándose en un manual diseñado a partir de experiencias de otros países (23), y b) el manejo concomitante, consistente y no violento del comportamiento de los niños que ya manifestaban problemas de comportamiento por parte de los padres. De esta manera, toda la población de niños participaría en el proyecto y aquellos niños con mayores problemas de comportamiento agresivo recibirían un apoyo adicional en sus hogares.

El diseño original preveía una serie de actividades a cargo de los maestros, a fin de que intervinieran en todas las situaciones de agresión, de manera concomitante, consistente y no violenta, y adquirieran el hábito de promover y modelar los comportamientos prosociales de los niños, y de reforzarlos cada vez que se presentaran. Simultáneamente con la intervención escolar, se preveía una intervención familiar, en la que un asesor familiar orientaría a los padres de los niños con síntomas de mayor agresividad en el análisis crítico de sus formas de interacción y comunicación con el niño y de sus técnicas de disciplina y supervisión, basándose en las actividades establecidas en una guía elaborada a partir de guías probadas en otros países y adaptada a las condiciones socioculturales de Medellín. En otra publicación, se detallan la orientación y los componentes de la intervención, tal como fue diseñada originalmente (24).

Una vez diseñado el programa, se consultaron de nuevo expertos de universidades locales y de la administración municipal para refinar el ajuste del diseño según las condiciones locales. Los expertos consultados consideraron que el modelo 
sociocognitivo que fundamentaba el diseño no era la orientación teórica más apropiada $\mathrm{y}$, por consiguiente, se reemplazó esta orientación por un enfoque psicodinámico y se reformularon los objetivos del programa para promover la reflexión sobre la manera en que los padres y los maestros proceden con los niños y desarrollan las prácticas de educación y crianza. También se varió el número de actividades previstas con los maestros y los padres. ${ }^{4}$

La primera fase del programa se llevó a cabo en el año 2001, según las características detalladas a continuación.

1. Se llevó a cabo en una de las zonas más pobres de la ciudad y con mayores problemas de marginalización y violencia.

2. Participaron niños de ambos sexos, de entre 3 y 11 años, escolarizados desde el grado 0 (jardín infantil) hasta el último grado de primaria (5. ${ }^{\circ}$ grado).

3. Ingresaron al programa las escuelas y los jardines infantiles públicos de la zona que aceptaron participar, y los maestros y jardineras infantiles de estas instituciones que lo solicitaron voluntariamente.

4. El trabajo con los maestros y las jardineras infantiles incluyó:

a. Capacitación por medio de diez talleres que se realizaron a lo largo de cuatro meses, basándose en un manual preparado para este programa. Los talleres abarcaron los siguientes temas:

- El papel del docente en el manejo de la violencia

- La escucha y la comunicación: fundamento de las habilidades sociales

- El respeto de la diferencia: brindado al niño una perspectiva de los demás

\footnotetext{
4 Agradecemos a la Profesora Eryka Montoya el habernos facilitado el documento de F. Domínguez "Observaciones al proceso de aplicación del modelo de prevención temprana de la agresión. Pautas de educación y crianza", en el cual se describen estos cambios
}

- La autoafirmación y la autoestima: ¿cómo expresar sanamente nuestros afectos?

- Promoviendo la cooperación entre los alumnos

- Le ética del maestro: el docente como modelos de valores.

- La administración del aula: ¿cómo convertirla en un lugar amable y funcional?

- La autoridad en el aula: sustentación, reconocimiento y negociación

- Ante la agresión: estrategias para el manejo del conflicto

- El docente y la violencia externa a la escuela

5. Talleres de asesoría a los maestros y jardineras, con una frecuencia de una sesión cada quince días durante 8 meses. La metodología utilizada fue diferente para el trabajo en escuelas y en jardines infantiles. En las escuelas, se realizaron talleres grupales con análisis de casos y se fomentó la expresión de sentimientos y emociones generadas en la interacción del maestro con el entorno y con el niño. En los jardines infantiles, se asesoró individualmente a las jardineras sobre el manejo de casos y las dinámicas del hogar.

a. Se llevó a cabo un trabajo con familias. Se ofreció apoyo a los padres de los niños con mayores síntomas de agresividad, seleccionados por medio de cribado con el cuestionario para la evaluación de comportamientos agresivos y prosociales (COPRAG, puntuación igual o superior a 90), diseñado y validado en Medellín (19). También se admitieron en el programa familias de niños que tenían una puntuación menor de 90 que solicitaron participar en él.

b. Se programaron una visita al domicilio de cada familia, seis talleres con los padres y tres sesiones de asesoramiento familiar. En los talleres se utilizó un manual de apoyo en el que se sugerían ocho temas, entre los que el asesor de familias elegía aspectos para profundizar en cada grupo, según el diagnóstico que previamente había realizado:

- La escucha y la comunicación: fundamento de las habilidades sociales

- El respeto de la diferencia: brindando al niño una perspectiva de los demás.

- La autoafirmación y la autoestima: ¿cómo expresar sanamente nuestros afectos?

- Promoviendo la cooperación en el hogar

- Los padres como modelos para el niño

- La autoridad en la familia: sustentación, reconocimiento y negociación

- Ante la agresión: estrategias para el manejo del conflicto

- La violencia externa al hogar y su manejo

6. La intervención duró doce meses, entre la capacitación de los maestros y la finalización de las actividades de apoyo a las familias. El seguimiento del programa se realizó durante un año y medio, con un enfoque cualitativo, utilizando técnicas como la observación participante, entrevistas semiestructuradas y análisis de diarios de campo.

7. Al final de la intervención, la adhesión de las familias que participaron en la primera etapa del programa fue:

a. alta (al menos uno de los padres asistió a por lo menos nueve de las diez sesiones de capacitación) en un $22 \%$ de los casos $(n=531)$,

b. aceptable (al menos uno de los padres asistió a seis, siete $\mathrm{u}$ ocho de las diez sesiones de capacitación) en un $21 \%$ de los casos $(\mathrm{n}=513)$,

c. baja (al menos uno de los padres asistió a cinco o menos sesiones de capacitación) en un 57\% de los casos $(n=1398)$.

8. La adhesión de los maestros que participaron en la primera etapa del programa fue:

a. alta (el maestro asistió a por lo menos nueve de las diez sesiones de capacitación) en un $58 \%$ de los casos $(\mathrm{n}=210)$, 
b. aceptable (el maestro asistió a ocho de las diez sesiones de capacitación) en un 5\% de los casos $(n=19)$,

c. baja (el maestro asistió a menos de ocho de las diez sesiones de capacitación) en un $37 \%$ de los $\operatorname{casos}(n=132)$.

En esta primera fase, que es la que hemos evaluado, se cubrieron 7605 niños de 33 instituciones públicas de enseñanza básica primaria y 24 guarderías públicas, y participaron 271 maestros, 164 jardineras y 2440 familias. En este momento, se está llevando a cabo la cuarta fase de expansión del Programa, que incluye, además, un elemento nuevo: la promoción de competencias ciudadanas.

Hasta donde llega nuestro conocimiento, esta experiencia es la de mayor envergadura en Colombia y, probablemente, en Latinoamérica, pues ha llegado a más de 90000 niños de los jardines infantiles y las escuelas públicas de Medellín, a través de cuatro etapas en las que se ha ido aumentando su cobertura entre 2001 y 2006.

El objetivo de este trabajo ha sido establecer el impacto de la fase inicial del Programa de Prevención Temprana de la Violencia - ejecutada por la Alcaldía de Medellín en 2001tres años después de la intervención, con respecto a comportamientos agresivos, conductas prosociales y rendimiento escolar.

\section{MATERIALES Y MÉTODOS}

Se llevó a cabo una comparación de mediciones realizadas en 2001 y 2004, es decir un análisis anterior y posterior a la intervención, en un grupo de niños incluidos en la primera fase del del Programa de Prevención Temprana de la Violencia, en 2001.

\section{Población}

La población estudiada estuvo compuesta por una muestra no probabilística de 339 niños de ambos sexos. Estos niños fueron todos los que se localizaron en 2004 de los 7605 que habían participado en la primera fase del programa, en 2001, tras una búsqueda cuidadosa en cuatro bases de datos que abarcaban: a) los niños evaluados con la prueba COPRAG (descrita más adelante) en 2001, b) las familias participantes en la primera fase del programa, c) los maestros y alumnos de las escuelas públicas y jardines infantiles de Medellín que participaron en el programa en 2001, y d) los maestros y alumnos de escuelas públicas de Medellín en 2004. Se identificaron los niños que figuraban en las cuatro bases de datos y, con la colaboración de la Secretaría de Educación y Cultura de Medellín, se realizó su búsqueda en las escuelas (a través de directores y maestros) y por medio de llamadas telefónicas y de visitas a los barrios donde era posible que residieran.

Al momento de la evaluación, la edad media de los niños en los que se realizó el estudio era de 11,8 años y la proporción de varones, del $68 \%$.

\section{Variables}

Las variables empleadas para medir los cambios eventualmente producidos por el programa fueron: a) síntomas de agresión directa, b) síntomas de agresión indirecta, c) síntomas de prosocialidad $\mathrm{y} d$ ) rendimiento escolar (cuadro 1).

\section{Medición}

El instrumento empleado para efectuar las mediciones fue la prueba COPRAG, que permite identificar síntomas de agresión directa (por ejemplo, atacar a otras personas, patear o morder a otros niños, participar en muchas peleas, destruir objetos de otros, mentir o hacer trampas) e indirecta (por ejemplo, cuando esta disgustado con alguien, busca que otros también lo estén, o se hace amigo de otros en venganza, o dice cosas en su contra a sus espaldas, o busca que los otros aíslen a esa persona) y síntomas de prosocialidad (por ejemplo, ayudar a alguien que se ha golpeado, intervenir para que no se produzca un alegato o discusión, o una pelea, ofrecer ayuda a otros cuando están en una dificultad), además de los síndromes de hiperactividad, déficit de atención, depresión, ansiedad y trastorno deficitario del aprendizaje. Este cuestionario, constituido de 50 ítems, se diseñó basándose en las pruebas utilizadas por Tremblay, Achenbach y Apodaca et al., y en el Manual diagnóstico y estadístico de los trastornos mentales (DSM-IV), y se probó en la población de Medellín, donde se encontraron altos índices de consistencia interna (19).

\section{Recolección de los datos}

Cada niño fue evaluado por su maestro, mediante la prueba COPRAG. Para ello, los maestros fueron previamente capacitados $y$ contaron con apoyo del personal de dirección del trabajo de campo de la investigación mientras llevaban a cabo las evaluaciones. Los maestros realizaron esta actividad al finalizar el año escolar, con el fin de tener un conocimiento adecuado de cada niño. Los encuestadores encargados de las entrevistas a los niños y a las familias también fueron capacitados para brindar acompañamiento y entrenamiento al maestro.

\section{Control de la calidad}

Para reducir las fuentes de error se realizó, de manera simultánea a la recolección, un proceso de control de datos faltantes o contradictorios, y una confirmación telefónica del 10\% de las encuestas.

\section{Análisis}

Como se observó una distribución asimétrica a la derecha de las variables, en las comparaciones se empleó la mediana y en el análisis 
CUADRO 1. Constructos de la prueba COPRAG (18) para la evaluación del Programa de Prevención Temprana de la Violencia de Medellín, Colombia, 2001 y 2004

\begin{tabular}{|c|c|}
\hline Variable & $\alpha$ de Cronbach \\
\hline $\begin{array}{l}\text { SÍNTOMAS DE AGRESIÓN DIRECTA } \\
\text { 1. Nunca, 2. Algunas veces y 3. Casi siempre } \\
\text { - Ataca físicamente a las personas } \\
\text { — Patea, muerde, golpea a otros niños } \\
\text { - Destruye (rompe, derriba o pisa voluntariamente) objetos que pertenecen } \\
\text { a su familia u otros niños } \\
\text { - Se mete en muchas peleas } \\
\text { - Es cruel, abusivo o malvado con otras personas o con los animales } \\
\text { — Daña juegos o actividades de los otros } \\
\text { - Cuando otro niño accidentalmente le golpea, asume que este quiso } \\
\text { hacerle daño y reacciona con rabia y pelea } \\
\text { — Es impulsivo, actúa sin pensar } \\
\text { - Toma objetos de otros niños sin su permiso. (Apropiación, ruptura de } \\
\text { objetos o juegos cuando un niño quita a otro - bien de su mano o de su } \\
\text { espacio- un objeto que esta usando y que no es ofrecido) } \\
\text { - Destruye sus propias cosas } \\
\text { — Amenaza con gestos o con palabras a los otros } \\
\text { - Tiene dificultades para esperar su turno en juegos o en grupos } \\
\text { - Dice mentiras, hace trampa } \\
\text { - Imita el llanto de otros niños }\end{array}$ & 0,93 \\
\hline $\begin{array}{l}\text { SÍNTOMAS DE AGRESIÓN INDIRECTA } \\
\text { 1. Nunca, 2. Algunas veces y 3. Casi siempre } \\
\text { - Cuando está disgustado con alguien le dice a los demás que no } \\
\text { compartan con esa persona } \\
\text { - Cuando está bravo con alguien, se hace amigo de otros como venganza } \\
\text { - Cuando está disgustado con alguien, dice a otros los secretos de éste } \\
\text { - Cuando está disgustado con alguien, dice cosas en su contra a sus } \\
\text { espaldas } \\
\text { - Cuando está bravo con alguien, intenta que otros hagan lo mismo con } \\
\text { esa persona } \\
\text { - Dice mentiras, hace trampa }\end{array}$ & 0,90 \\
\hline 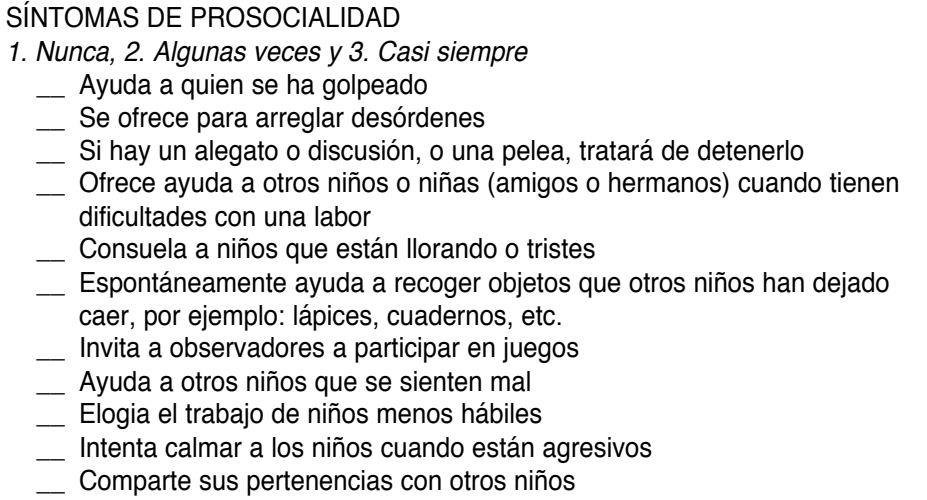 & 0,90 \\
\hline
\end{tabular}

bifactorial, la prueba no paramétrica de la mediana para muestras independientes o la prueba de Wilcoxon para muestras equiparadas. En el análisis multifactorial, se empleó el análisis de regresión logística condicional para muestras pareadas $(25,26)$, para obtener la razón de posibilidades (odds ratio) y su significación estadística (27).

Los constructos de la prueba COPRAG utilizados en este estudio (síntomas de agresión directa, de

\section{RESULTADOS}

agresión indirecta y de prosocialidad) se crearon a partir de análisis factorial y presentaron en esta muestra un alto grado de consistencia interna. El $\alpha$ de Cronbach varió entre 0,90 y 0,93 (cuadro 1).

En el cuadro 2 se comparan los valores de las variables de impacto determinados antes (2001) y después (2004) de la primera fase del programa, expresados como la mediana de las puntuaciones factoriales o el porcentaje. Se observó una disminución estadísticamente significativa en los síntomas de agresión directa, de $-0,07$ (intervalo de confianza del 95\% (IC95\%): $-0,211-0,086$ ) a $-0,272$ (IC95\%: $-0,403-$ $-0,189)$, y de agresión indirecta, de 0,343 (IC95\%: 0,101-0,445) a -0,316 (IC95\%: $-0,532--0,088)$, y un aumento en los síntomas de prosocialidad, de $-0,026$ (IC95\%: -0,164 - 0,092) a 0,26 (IC95\%: $0,13-0,345)$. Adicionalmente hubo un aumento del porcentaje de niños con alto rendimiento escolar, del 11,0\% (IC95\%: $7,6 \%-14,4 \%$ ) al $20,3 \%$ (IC95\%: $16,0 \%$ $24,6 \%$ ), y una disminución del porcentaje de niños con resultados inferiores al promedio $(P<0,01)$.

Con el fin de estimar si estos cambios están relacionados con el sexo, la edad y el nivel de agresión de los niños, y con el componente de apoyo a las familias, se realizó un análisis de regresión logística por cada una de estas variables (cuadro 3). Se halló una asociación entre el grado de adhesión de la familia al programa y el incremento de los síntomas de prosocialidad y la disminución de los síntomas de agresión indirecta, puesto que estas diferencias sólo se observaron entre los niños de familias con alta adhesión al programa.

La disminución de los síntomas de agresión directa se observó entre los niños con mayor grado de agresión, pero no estuvo relacionada con el sexo, ni con la edad. La disminución de los síntomas de agresión indirecta fue estadísticamente significativa en las niñas, pero no en los niños, y en los mayores de 12 años, pero no en los menores de 12 años y entre quienes presentaban mayor puntuación de síntomas de agresión. El aumento de la prosocialidad fue significativo en ambos sexos, en todas las edades estudiadas y 
CUADRO 2. Evaluación de los síntomas de agresión y del rendimiento escolar en 2001 y 2004 de 335 niños participantes en el Programa de Prevención Temprana de la Violencia, primera etapa. Medellín, Colombia, 2001

\begin{tabular}{|c|c|c|c|c|c|}
\hline Variable & Unidad o categoría & Unidad de medida & $2004^{a}$ & $2001^{a}$ & $P$ \\
\hline Síntomas de prosocialidad & $\begin{array}{l}\text { Puntuación en la } \\
\text { prueba COPRAG }\end{array}$ & Mediana & $\begin{array}{c}0,26 \\
(0,13-0,34)\end{array}$ & $\begin{array}{c}-0,03 \\
(-0,16-0,09)\end{array}$ & 0,000 \\
\hline
\end{tabular}

a Se indican entre paréntesis los intervalos de confianza del $95 \%$.

CUADRO 3. Razón de disparidada ${ }^{a}$, modelo de regresión logística condicional ${ }^{b}$, de las mediciones de las variables estudiadas realizadas en 2004 con respecto de las mediciones de 2001, según la adhesión de la familia al programa, el sexo, la edad y el nivel de agresión del niño. Evaluación del Programa de Prevención Temprana de la Violencia (primera etapa), Medellín, Colombia.

\begin{tabular}{|c|c|c|c|c|c|c|c|c|c|}
\hline \multirow[b]{2}{*}{ Variable } & \multicolumn{3}{|c|}{ Adhesión de la familia al programa } & \multicolumn{2}{|c|}{ Sexo } & \multicolumn{2}{|c|}{ Edad } & \multicolumn{2}{|c|}{$\begin{array}{l}\text { Nivel de agresión } \\
\text { (COPRAG) }\end{array}$} \\
\hline & General & Alta & Baja & Varones & Mujeres & $\leq 12$ Años & > 12 Años & Bajo & Alto \\
\hline $\begin{array}{l}\text { Síntomas de agresión } \\
\text { directa }\end{array}$ & $\begin{array}{c}0,81 \\
(0,68-0,96)\end{array}$ & $\begin{array}{c}0,86 \\
(0,64-1,15)\end{array}$ & $\begin{array}{c}0,74 \\
(0,57-0,96)\end{array}$ & $\begin{array}{c}080 \\
(0,65-0,98)\end{array}$ & $\begin{array}{c}0,82 \\
(0,57-1,17)\end{array}$ & $\begin{array}{c}0,85 \\
(0,63-1,14)\end{array}$ & $\begin{array}{c}0,79 \\
(0,64-0,98)\end{array}$ & $\begin{array}{c}1,03 \\
(0,82-1,30)\end{array}$ & $\begin{array}{c}0,44 \\
(0,31-0,64)\end{array}$ \\
\hline $\begin{array}{l}\text { Síntomas de agresión } \\
\text { indirecta }\end{array}$ & $\begin{array}{c}0,70 \\
(0,59-0,82)\end{array}$ & $\begin{array}{c}0,60 \\
(0,45-0,80)\end{array}$ & $\begin{array}{c}0,77 \\
(0,60-0,98)\end{array}$ & $\begin{array}{c}0,84 \\
(0,68-1,03)\end{array}$ & $\begin{array}{c}0,47 \\
(0,35-0,65)\end{array}$ & $\begin{array}{c}0,85 \\
(0,63-1,13)\end{array}$ & $\begin{array}{c}0,63 \\
(0,51-0,77)\end{array}$ & $\begin{array}{c}0,79 \\
(0,64-0,96)\end{array}$ & $\begin{array}{c}0,41 \\
(0,29-0,60)\end{array}$ \\
\hline Síntomas de prosocialidad & $\begin{array}{c}1,52 \\
(1,28-1,80)\end{array}$ & $\begin{array}{c}1,92 \\
(1,43-2,57)\end{array}$ & $\begin{array}{c}1,28 \\
(1,00-1,63)\end{array}$ & $\begin{array}{c}1,42 \\
(1,17-1,73)\end{array}$ & $\begin{array}{c}1,74 \\
(1,24-2,44)\end{array}$ & $\begin{array}{c}1,40 \\
(1,04-1,88)\end{array}$ & $\begin{array}{c}1,58 \\
(1,29-1,93)\end{array}$ & $\begin{array}{c}1,44 \\
(1,19-1,75)\end{array}$ & $\begin{array}{c}1,47 \\
(1,00-2,16)\end{array}$ \\
\hline $\begin{array}{l}\text { Rendimiento escolar similar } \\
\text { al de los compañeros } \\
\text { de la misma edad }\end{array}$ & $\begin{array}{c}0,90 \\
(0,74-1,10)\end{array}$ & $\begin{array}{c}0,79 \\
(0,55-1,12)\end{array}$ & $\begin{array}{c}0,88 \\
(0,65-1,19)\end{array}$ & $\begin{array}{c}0,91 \\
(0,71-1,16)\end{array}$ & $\begin{array}{c}1,10 \\
(0,71-1,69)\end{array}$ & $\begin{array}{c}0,82 \\
(0,56-1,19)\end{array}$ & $\begin{array}{c}0,95 \\
(0,75-1,21)\end{array}$ & $\begin{array}{c}0,93 \\
(0,74-1,18)\end{array}$ & $\begin{array}{c}1,76 \\
(1,02-3,03)\end{array}$ \\
\hline $\begin{array}{l}\text { Rendimiento escolar supe- } \\
\text { rior al de los compañeros } \\
\text { de la misma edad }\end{array}$ & $\begin{array}{c}2,20 \\
(1,43-3,39)\end{array}$ & $\begin{array}{c}1,38 \\
(0,67-2,84)\end{array}$ & $\begin{array}{c}3,70 \\
(1,91-7,20)\end{array}$ & $\begin{array}{c}2,17 \\
(1,31-5,59)\end{array}$ & $\begin{array}{c}2,53 \\
(0,98-6,50)\end{array}$ & $\begin{array}{c}1,94 \\
(0,93-4,03)\end{array}$ & $\begin{array}{c}2,31 \\
(1,35-3,97)\end{array}$ & $\begin{array}{c}1,68 \\
(1,04-2,72)\end{array}$ & $\begin{array}{c}11,76 \\
(3,86-35,71)\end{array}$ \\
\hline
\end{tabular}

a Razón de disparidad (odds ratio) 2004-2001; se indican entre paréntesis los intervalos de confianza del 95\%.

${ }^{\mathrm{b}}$ La ecuación del modelo de regresión logística utilizado es la siguiente:

$$
\operatorname{LnRD}=\operatorname{Ln}\left[\frac{Y_{j}}{1-Y_{i}}\right]=\sum_{j=0}^{5} \beta_{j} X_{j}
$$

En donde $L n R D$ es el logaritmo natural de la razón de disparidad, $Y_{i}$, el valor de la variable según el año de medición $\left(Y_{i}=1\right.$ en 2004 y $Y_{i}=0$ en 2001$)$, $X_{0} X_{1}, \ldots, X_{5}$ son, respectivamente, las intersecciones (en este caso $=1$ ), síntomas de agresión directa, síntomas de agresión indirecta y comportamiento prosocial, y las variables de rendimiento escolar similar o superior al de los demás niños de la misma edad, y $\beta_{, j}$, la estimación del efecto producido por las variables independientes $X_{j}$.

entre quienes presentaban menor nivel de agresión

La mejora del rendimiento escolar con respecto a niños de la misma edad se observó en los mayores de 12 anos y fue de mayor magnitud entre los niños con mayor puntuación de agresión. Paradójicamente, se asoció con baja adhesión al programa por parte de la familia.

\section{DISCUSIÓN}

Antes de proceder a una discusión de los resultados obtenidos, es conveniente indicar las limitaciones que presenta este estudio. Los integrantes de la muestra estudiada fueron los niños que se localizaron después de una intensa y larga búsqueda, que duró cerca de un año; es posible que estos niños hayan sido aquellos con menos estresores en el ambiente en que vivían y que se haya dejado fuera a los que cambiaron de residencia por temor a la violencia en el barrio o por otras razones. $\mathrm{La}$ falta de una comparación con un grupo de referencia no permite descartar explicaciones alternativas, como la maduración, los factores externos a la 
intervención o el impacto de la medición. La falta de mediciones inmediatamente posteriores a la finalización de la intervención no permitió establecer el impacto inmediato. Sin embargo, la realización de una evaluación a los tres años de la intervención proporciona información sobre el impacto potencial del programa a más largo plazo; este dato es de mayor interés, puesto que en algunos casos, las intervenciones tempranas empiezan a mostrar sus efectos sólo después de tres años o más de iniciadas (28).

No obstante, cabe destacar dos puntos fuertes de este estudio. En primer lugar, es importante disponer de información sobre la evaluación de programas de prevención temprana de la violencia en un país en vías de desarrollo, dado que la mayoría de estos estudios se ha llevado a cabo en países desarrollados. En segundolugar, se trata de la evaluación de un programa llevado a cabo en la comunidad a gran escala, que ha tenido tres etapas de expansión a otras escuelas en años posteriores, y no de un proyecto piloto o de demostración realizado en condiciones controladas, como la mayoría de los programas de prevención temprana de la violencia cuyo efecto se ha evaluado (29).

Hay varios factores que contribuyen a explicar por qué los efectos positivos asociados al programa no se presentan por igual por sexo, edad, nivel de agresión del niño y grado de adhesión de la familia al programa. El programa sufrió importantes modificaciones con respecto al diseño inicial. El cambio de fundamentación teórica sobre la marcha puede haber conducido a definir, en la primera etapa, un programa sin coherencia, ni sociocognitivo, ni psicoanalítico, sino compuesto de elementos provenientes de ambas corrientes de pensamiento. Como se ha indicado antes, la fundamentación teórica sólida y coherente es una de las condiciones básicas para el éxito de una intervención de prevención temprana de la agresión. Se ha referido que no existe suficiente evidencia empírica sobre el impacto de los programas de enfoque psicoanalítico en la prevención de la violencia (13). Esta evaluación puede ser un paso para documentar efectos de una intervención que tuvo un contenido mixto -psicodinámico y sociocognitivo- y va a ser muy interesante comparar sus resultados con los de las evaluaciones de las etapas siguientes del programa, en las cuales se ha acentuado la orientación psicodinámica. El cambio de enfoque pudo haber influido en la forma como finalmente se implementó el programa. Según información suministrada por la encargada del seguimiento del programa, los análisis cualitativos de los diarios de campo sugieren que los maestros cambiaron su manera de pensar acerca de cómo manejar los comportamientos agresivos en los niños y cómo intervenir en situaciones de conflicto, pero no sabemos si modificaron su forma de actuar.

La disminución de la frecuencia de las actividades originalmente programadas también pudo haber influido en los resultados. Según los informes de supervisión, la situación de alta peligrosidad que se presentaba en el vecindario donde se llevó a cabo la intervención y la ausencia de los padres en horas diurnas fueron dos de las razones por las cuales se disminuyó el número de visitas domiciliarias. Por otra parte, aunque se realizó un seguimiento cualitativo de los maestros, no se les siguió proporcionando un refuerzo suficiente después de terminada su capacitación. Además, por lo general, a cada sesión de actividades con la familia acudían diferentes miembros del grupo familiar. Diversos autores han resaltado la importancia de establecer una «dosis» adecuada de las acciones como un elemento básico para el buen éxito de los programas de prevención de la violencia (18). Asimismo se ha señalado que los programas de corta duración (menos de un año) tienen efectos limitados en el tiempo, mientras que los de mayor duración tienen efectos más permanentes en la vida $(30,31)$.

La baja adhesión al programa es otro elemento que puede haber influido en el resultado obtenido. En un programa similar, se ha comunicado una adhesión del $87 \%$ entre los maestros $(32,33)$, mientras que en el programa de Medellín, esta adhesión fue del 58\%. Este valor relativamente bajo puede deberse al hecho de que, a diferencia de los jardines infantiles, donde todas las jardineras y el personal administrativo y de servicios fueron capacitados y recibieron importante apoyo de los directores, en las escuelas, sólo se capacitó a los maestros que quisieron participar voluntariamente en el programa, por lo que en varias de ellas sólo se aplicó el programa en una o dos clases. Esta baja concentración por escuela puede haber influido en el resultado del programa. En cuanto a la adhesión de las familias, la proporción llamativamente baja en Medellín (57\%) es bastante similar a la referida en la experiencia citada $(47 \%)$.

La información obtenida en esta evaluación indica que esta experiencia parece haber tenido mejores resultados en el aumento de las conductas prosociales que en la disminución de la agresión, dado que el aumento de la prosocialidad se presentó entre los niños cuyas familias tuvieron una alta adhesión al programa y no entre quienes tuvieron muy baja; en el sexo, aunque más en las mujeres y en ambos grupos de edad estudiados. Cabe resaltar que el incremento de la prosocialidad se observó entre los niños menos agresivos, lo cual sugiere que la intervención refuerza conductas positivas entre aquellos que tienen menores problemas de comportamiento.

El efecto del programa en los síntomas de agresión directa tiene lugar en los niños más agresivos, en los que se observa una disminución de casi un 50\%, lo que indica un gran potencial para la prevención de la agresión directa en este grupo, al que pertenece un bajo porcentaje de la comunidad de niños y jóvenes, que tiene, sin embargo, destacada importancia en el fenómeno de agresión en el conglomerado infantil y juvenil $(11,12,34)$.

También es de resaltar que, como la disminución de la agresión indirecta tuvo lugar en las niñas con más altos niveles de síntomas agresivos, en las que se obtuvo una disminución del $50 \%$, es posible que esta intervención tenga un impacto importante en la comunidad de escolares, dado que este tipo de agresión se presenta principalmente en las niñas, según estudios realizados en Colombia y en otros países $(35,36)$.

El apoyo a las familias parece haber tenido un impacto incremental sobre los efectos del otro componente del programa, centrado en la escuela, en lo 
referente a la disminución de la agresión indirecta $y$ el incremento de la prosocialidad, pero no en la disminución de la agresión directa, ni en el rendimiento escolar, que, como se indicó antes, no varió.

El análisis anterior y posterior, como el realizado en esta evaluación, presenta limitaciones para extraer conclusiones sobre si los cambios observados pueden atribuirse o no al programa evaluado. Por ello estamos concluyendo un análisis basado en el seguimiento durante tres años de estos niños, en comparación con un grupo de referencia.

A pesar de estas limitaciones y de las dificultades que se presentaron en la implementación de la primera fase de este programa, el presente informe aporta elementos importantes en el campo de la prevención de violencia en Latinoamérica, dada la escasez de publicaciones sobre la evaluación de programas de prevención temprana de la violencia en esta región y, en especial, de programas que no sean de demostración, sino de cobertura amplia en la comunidad.

Hay que destacar los esfuerzos de la municipalidad de Medellín, comprometida desde varios años con importantes inversiones en el desarrollo de políticas públicas y programas de prevención de la agresión desde la infancia. Dado que los resultados de la etapa inicial del Programa de prevención temprana de la violencia son interesantes, creemos que este esfuerzo de tratar de prevenir la violencia desde la infancia debe continuarse por largos años. Consideramos que deben introducirse ajustes en el programa, basados en la presente evaluación y en otras que se están llevando a cabo sobre algunas de las etapas posteriores.

\section{REFERENCIAS}

1. Organización Mundial de la Salud. World Health Report 2002. Statistical Annex. [Sitio en Internet] WHO. Hallado en: http:// www.who.int/whr/2002/en/whr02_en.pdf. Acceso en agosto 23 de 2004.

2. Colombia, Ministerio de Salud. La carga de la enfermedad en Colombia. Santa Fe de Bogotá: Ministerio de Salud; 2000.

3. Londoño JL, Grisales H, Fernández SJ, Cadena E. Años de vida saludable perdidos por la población de Medellín. Un análisis especial por homicidio y accidentes de vehículo motor. Rev Fac Nac Sal Publ. 1999;17(1):63-92

4. Connor DF. Aggression and antisocial behavior in children and adolescents: research and treatment. New York: The Guilford Press; 2002. Pp. 46-61.

5. Olweus D. Stability of aggressive reaction patterns in males. A review. Psychol Bull. 1979; 86(4):852-75

6. Loeber R. The stability of antisocial and delinquent child behaviour: a review. Child Dev. 1982;53:1431-46.

7. Nagin D, Tremblay R.E. Trajectories of boys' physical aggression, opposition, and hyperactivity on the path to physically violent and nonviolent juvenile delinquency. Child Dev. 1999;70:1181-96.

8. Keenan K, Shaw DS. The development of aggression in toddlers: A study of low income families. J Abnorm Child Psychol. 1994;22: 53-77.

9. Brame B, Nagin DS, Tremblay RE. Developmental trajectories of physical aggression from school entry to late adolescence. J Child Psychol Psychiatry. 2001;42:503-12.
10. White, JL, Moffitt TE, Earls F, Robins L, Silva PA. How early can we tell?: Predictors of childhood conduct disorder and adolescent delinquency. Criminology. 1990;28:507-32.

11. Farrington DP. Key results from the first forty years of the Cambridge study in delinquent development. En: Thornberry T, Krohn MD, eds. Taking stock of delinquency. An overview of findings from contemporary longitudinal studies. New York: Kluwer Academic/ Plenum Publishers; 2003. Pp. 137-79.

12. Loeber R, Farrington DP, Stouthamer-Loeber M, Moffitt T, Caspi A. The development of Male Offending. Key Findings from Fourteen Years of the Pittsburgh Youth Study. En: Thornberry T, Krohn MD, eds. Taking stock of delinquency. An overview of findings from contemporary longitudinal studies. New York: Kluwer Academic/Plenum Publishers; 2003. Pp. 96-136.

13. Huizinga D, Weiher A, Espiritu R. Delincuency and crime: some highlights from the Denver Youth Survey. En: Thornberry T, Krohn MD, eds. Taking stock of delinquency. An overview of findings from contemporary longitudinal studies. New York: Kluwer Academic/Plenum Publishers; 2003. Pp. 156-7

14. Connor DF. Aggression and antisocial behavior in children and adolescents: research and treatment. New York: The Guilford Press; 2002. Pp. 62-112. contemporaneous co-occurrence of serious and violent juvenile offending and other problem behaviours. En: Loeber R, Farrington DP, eds. Seriuos \& violent juvenile offenders.
15. Huizinga D, Jacob Chien C. The
Tal vez la principal recomendación que surge de este trabajo es la importancia de ensayar y evaluar los nuevos programas primero a pequeña escala. Es buena costumbre en el ámbito de la salud pública establecer la eficacia de un programa (el impacto de la intervención cuando se implementa correctamente y todos lo reciben en la dosis recomendada) antes de su efectividad (el impacto entre aquellos en los que se ofrece con sus diferentes grados de adhesión), previamente a la aplicación a gran escala. La realización de dos proyectos pilotos del Programa de prevención temprana de la violencia, uno según el diseño original y otro según el diseño modificado, hubiera permitido identificar rápidamente los problemas en la implementación, así como ahorrar recursos al establecer la efectividad de cada uno en una población de menor tamaño.
Thousand Oaks: Sage Publications; 1999. Pp. 47-67.

16. National Research Council and Institute of Medicine. From Neurons to Neighborhoods: The science of early childhood development. Committee on integrating the science of early childhood development. Jack P. Shonkoff Deborah A. Philips (eds). Board on Children, Youth, and Families, Commission on Behavioral and Social Sciences and Education. Washington DC.: National Academy Press. Pp. 337-380

17. Blueprints for Violence Prevention Overview. [Sitio en internet] University of Colorado at Boulder, Institute of Behavioral Science, Center for the Study and Prevention of Violence. Hallado en: http:/ / www.colorado.edu/cspv/ blueprints/index.html. Acceso el 8 de noviembre de 2004.

18. National Institutes of Health State-of-the-Science Conference Statement. Preventing Violence and Related Health-Risking Social Behaviors in Adolescents: an NIH State-of-the-Science Conference; 2004 oct.; Bethesda, Estados Unidos. Bethesda (MD): NIH; 2004

19. Agudelo LM, Giraldo CA, Gaviria MB, Sandoval CA, Rodriguez MA, Gómez JF et al Características de las familias y escuelas relacionadas con los comportamientos agresivos y prosociales en niños y niñas de 3-11 años. Colciencias, CES y Universidad de Antioquia. Medellin: Marín Vieco; 2002.

20. Campbell SB. Behavior problems in preschool children: A review of recent research. J Child Psychol Psychiatry. 1995;36:113-49.

21. Kratzer L, Hodgins S. Adult outcomes of child conduct problems: A cohort study. J Abnorm Child Psychol. 1997;25(1):65-81. 
22. Duque LF. El Programa de Convivencia Ciudadana de Medellín. Medellín: Alcaldía de Medellín; 2000.

23. Slaby RG, Roedell WC, Arezzo D, Hendrix K. Early violence prevention: Tools for teachers of young children. Washington, D.C.: National Association for the Education of Young Children; 1995.

24. Duque LF, Klevens J, Ungar M, Lee AW. Violence Prevention Programming in Colombia: Challenges in Project Design and Fidelity. En: Ungar M, ed. A Handbook for working with children and youth: Pathways to resilience across cultures and contexts. Thousand Oaks (CA): SAGE Publications; 2005. Pp. 455-71.

25. Hosmer DW, Lemeshow S. Applied Logistic Regression. 2.a ed. New York: John Wiley and Sons; 2000.

26. Londoño JL. Metodología de la investigación epidemiológica. Bogotá: Manual Moderno; 2004.

27. Campbell DT, Stanley JC. Experimental and quasi-experimental designs for research. Chicago: Rand McNally; 1963.

28. Tremblay R, Vitaro F, Nagin D, Pagani L, Séguin JR. The Montreal longitudinal experimental study: Rediscovering the power of descriptions. En: Thornberry TP, Krohn MD, eds. Taking stock of delinquency. An overview of findings from contemporary longitudinal studies. New York: Kluwer Academic/ Plenum Publishers; 2003. Pp 205-41.

29. Lerner R, Fisher CB, Weinberg RA. Toward a science for and of the people: Promoting civil society through the application of developmental science. Child Dev. 2000;71:11-20

30. Eckenrode J, Ganzel B, Henderson CR Jr, Smith E, Olds DL, Powers J, et al Preventing child abuse and neglect with a program of nurse home visitation: The limiting effects of domestic violence. JAMA. 2000;284(11): 1385-91.

31. Blueprints Promising Programs. I Can Problem Solve (ICPS). [Sitio en internet] University of Colorado at Boulder, Institute of Behavioral Science, Center for the Study and Prevention of Violence. Hallado en: http:/ / www.colorado.edu/cspv/blueprints / promising/programs/BPP08.html. Acceso en 29 de septiembre de 2004.

32. Tremblay RE, Pagani K, Mâsse LC, Vitaro F, Pihl RO. A bimodal preventive intervention for disruptive kindergarten boys: Its impact through mid-adolescence. J Consult Clin Psychol. 1995;63:560-8.

33. Vitaro F, Brendgen $M$, Tremblay RE. Prevention of school dropout through the reduction of disruptive behaviors and school failure in elementary school. J Sch Psychol. 1999;37: 205-26.

34. Broidy LM, Nagin DS, Tremblay RE, Bates JE Brame B, Dodge K, et al. Developmental trajectories of childhood disruptive behaviours and adolescent delinquency: A six site, cross national study. Dev Psychol. 2003;39(2): 222-45

35. Archer J, Coté S. Sex difference in Aggressive Behavior: A Developmental and Evolutionary Perspective. En: Tremblay R, Hartup W., Archer J, eds. Developmental origins of aggression. New York: The Guildford Press; 2005. Pp. 429-34.

36. Gorman-Smith D, Loeber R. Are developmental pathways in disruptive behaviors the same for girls and boys? J Child Fam Stud. 2005; 14(1):15-27.

Manuscrito recibido el 19 de octubre de 2005. Aceptado para publicación, tras revisión, el 30 de noviembre de 2006

ABSTRACT Objective. To describe the components and development of the Early Prevention of Violence Program in the city of Medellín, Colombia, and to evaluate the results of its first phase, three years after implementation.

\section{Lessons learned from an early intervention violence prevention program in Medellín, Colombia} Methods. A before (2001) and after (2004) study of four variables-direct aggression, indirect aggression, prosocial behavior, and scholastic achievementwas conducted among a convenience sample of 339 program participants and their families.

Results. Several program benefits were noted. Decreases in both direct and indirect aggression were observed, though the latter was reduced only in girls and in those over 12 years old. Prosocial behavior increased among children of all ages and both genders, including those who exhibited low levels of prosocial behavior in 2001. In addition, improved school performance was seen in the group as a whole. Results may have been affected by some changes to the prevention program's implementation and by the dangerous nature of the neighborhood, which limited the home visits.

Conclusions. The program seems to be an effective intervention for highly aggressive children, among whom a decline in direct aggression was observed. It also offers preventive benefits, as evidenced by the rise of prosocial behaviors in less aggressive children.

Key words Violence, aggression, underachievement, program evaluation, Colombia. 